\title{
Dilute-Solution Behavior of Cellulose Tris(3,5-dimethylphenylcarbamate)
}

\author{
Akihiko TsuboI, Mikiya YamasaKi, Takashi NorisuYe, \\ and Akio Teramoto \\ Department of Macromolecular Science, Osaka University, \\ Toyonaka, Osaka 560, Japan
}

(Received July 5, 1995)

\begin{abstract}
Eleven samples of cellulose tris(3,5-dimethylphenylcarbamate) ranging in weight-average molecular weight $M_{w}$ from $2 \times 10^{4}$ to $4 \times 10^{6}$ have been prepared by extensive fractionation and studied by static light scattering, sedimentation equilibrium, and viscometry with 1-methyl-2-pyrrolidone at $25^{\circ} \mathrm{C}$ as the solvent. This polymer is found to exhibit pronounced optical anisotropy differing from other cellulose derivatives, and light scattering data are corrected for the anisotropy effect on the basis of the Nagai theory for the Kratky-Porod wormlike chain with cylindrically symmetric polarizabilities. It is shown that the molecular weight dependences of $\left\langle S^{2}\right\rangle_{z}$ (the $z$-average mean-square radius of gyration), $\delta$ (the optical anisotropy factor), and [ $\eta$ ] (the intrinsic viscosity) for $M_{w}$ below $10^{6}$ are explained consistently by the known theories for the unperturbed wormlike chain. From the comparison between theory and experiment the persistence length and the monomeric length along the chain contour are estimated to be 7.8 and $0.52 \mathrm{~nm}$, respectively. It is also shown that excluded-volume effects on $\left\langle S^{2}\right\rangle_{z}$ and $[\eta]$ are experimentally visible for $M_{w}$ above $10^{6}$ but remain small even at the highest $M_{w}$ studied.

KEY WORDS Cellulose Tris(3,5-dimethylphenylcarbamate) / Cellulose Derivative / Wormlike Chain / Persistence Length / Optical Anisotropy / Radius of Gyration / Intrinsic Viscosity /
\end{abstract}

Cellulose triphenylcarbamate derivatives, when used for stationary phases in highperformance liquid chromatography, show chiral recognition for racemic compounds, allowing efficient chromatographic separation of them. ${ }^{1-4}$ This optical resolution capacity depends on the kind of substituent, the number of substituents, and their positions on the phenyl group, and the highest efficiency is attained by the 3,5-dimethyl-substituted derivative, ${ }^{2,4}$ i.e., cellulose tris(3,5-dimethylphenylcarbamate) (CTDC) whose chemical structure is shown in Figure 1. Despite this remarkable capacity of CTDC, little information on its physical properties is yet available in the literature. The present study was commenced to determine molecular characteristics of CTDC fractions in dilute solution, with primary interest in estimating the stiffness of the polymer chain.

At an early stage of this work, we found that CTDC is soluble in 1-methyl-2-pyrrolidone (or $\mathrm{N}$-methyl-2-pyrrolidone, NMP) at room temperature but not in common organic solvents. This solubility feature is distinct from that of cellulose tris(phenylcarbamate), which dissolves in a number of common solvents. ${ }^{5-8}$ The lack of common solvents is of great advantage to wide use of CTDC for stationary phases in liquid chromatography but definitely disadvantageous to the solution characterization. Though we searched for other solvents for CTDC, the solubility test ended up with finding a few solvents (acetophenone, benzophenone, and dimethyl sulfoxide at elevated temperature) that were all useless for the 
preparation of CTDC fractions with different molecular weights. Thus, with NMP as the solvent, we fractionated CTDC samples and carried out light scattering, sedimentation equilibrium, and viscosity measurements on 11 selected fractions ranging in molecular weight from $2 \times 10^{4}$ to $4 \times 10^{6}$. The experimental results obtained are presented below, along with their analyses based on the wormlike chain model. ${ }^{9}$

\section{EXPERIMENTAL}

\section{Samples}

Two commercially available samples of cellulose, A (Avicel) and C (cotton linters), and 3,5-dimethylphenylisocyanate (DPI) supplied by Daicel Co. were used for the preparation of CTDC. Cellulose $\mathrm{C}$ was purified by washing first with aqueous sodium hydroxide containing a detergent and hydrogen peroxide, then thoroughly with water (seven times), and finally with methanol and benzene (each twice), while cellulose A was washed only with methanol and benzene. The DPI was used without further purification. The viscosityaverage molecular weights of celluloses $\mathrm{A}$ and $C$ were $2.7 \times 10^{4}$ and $3 \times 10^{5}$, respectively, when estimated with Henry's intrinsic viscositymolecular weight relation in cadoxen. ${ }^{10}$ The cadoxen used was made according to Henry's method. ${ }^{10}$ CTDC was prepared in the following way.

Cellulose (A or C vacuum-dried at $60^{\circ} \mathrm{C}$ for $24 \mathrm{~h}$ just before use) was allowed to react with DPI in pyridine at $140-150^{\circ} \mathrm{C}$ under nitrogen atmosphere. The molar ratio of DPI to glucose was adjusted to 9, i.e., three times that expected for the fully substituted derivative. When the reaction mixture became clear brown, the product was precipitated into a large quantity of methanol and collected on a glass filter. It was reprecipitated from an NMP solution into methanol after the solution had been centrifuged at $15000 \mathrm{rpm}$ for $40 \mathrm{~min}$ to remove insoluble part. Reprecipitation was then re-

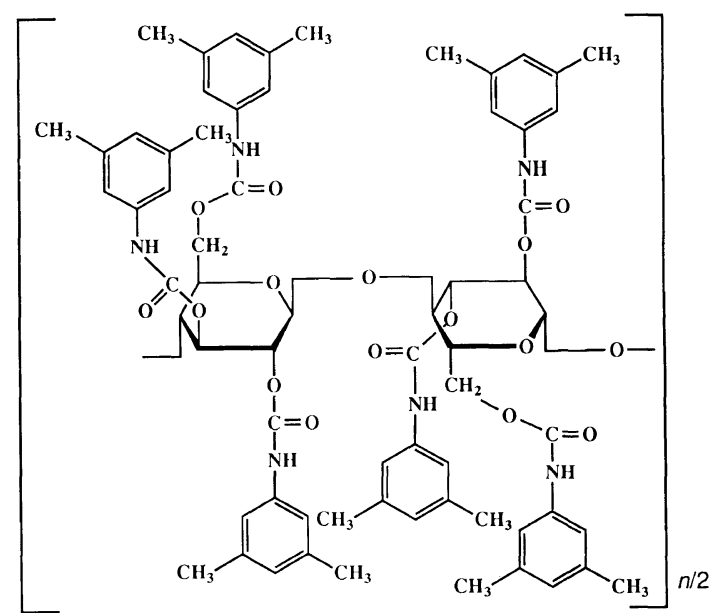

Figure 1. Chemical structure of CTDC.

peated five times with NMP containing $48 \%$ acetone as the solvent and aqueous 50\% methanol as the precipitant, and finally the polymer was dried in vacuo for 6 days. Its degree of substitution was found to be 17$30 \%$ lower than the expected value 3 when examined by elementary analysis. Thus, the above entire procedure was repeated once more with the reacted polymer, but the DPI-glucose molar ratio was adjusted to 3 this time. Elementary analysis showed that the products thus obtained from celluloses $\mathrm{A}$ and $\mathrm{C}$ were fully substituted. Their intrinsic viscosities [ $\eta]$ in NMP at $25^{\circ} \mathrm{C}$ were $0.65 \times 10^{2} \mathrm{~cm}^{3} \mathrm{~g}^{-1}$ (from cellulose A) and $2.2 \times 10^{2} \mathrm{~cm}^{3} \mathrm{~g}^{-1}$ (from cellulose C).

The CTDC samples were fractionated with NMP as the solvent and methanol as the precipitant. To an NMP solution of each sample with a polymer concentration of $0.5-1 \%$ the precipitant was slowly added at a fixed temperature of 20 or $25^{\circ} \mathrm{C}$ under stirring until the solution became turbid. The turbidity disappeared when the solution was warmed to $40-65^{\circ} \mathrm{C}$. It was then allowed to become cloudy again by cooling to the original temperature and kept standing overnight. However, the turbid solution did not completely separate into two phases. Thus the precipitate 
was collected on a glass filter in a temperatureregulated filtration apparatus, followed by reprecipitaion from an NMP solution (containing acetone) into aqueous methanol. The filtrate was subjected to further fractionation. In this way, each CTDC sample was divided into several parts. Middle fractions were white and powdery.

By repeating the above fractionation procedure three to six times, about 60 products were extracted. In some cases but not in the final fractionation step, fractions having nearly identical intrinsic viscosities were combined to minimize the loss of samples. It was confirmed by elementary analysis for arbitrarily chosen middle fractions that the degree of substitution did not vary with $[\eta]$. From the CTDC fractions thus prepared, 11 middle ones designated below as M4b, M2, T940, T580, T360, T170, T100, T66, T38, T29, and T22 were chosen for the present study.

\section{Light Scattering}

Total intensity measurements on NMP solutions of CTDC were made at $25^{\circ} \mathrm{C}$ on a Fica 50 light scattering photometer in an angular range from 30 to $150^{\circ}$, using vertically polarized incident light of 436 or $546-\mathrm{nm}$ wavelength. In all actual experiments for the former wavelength, however, a 436-nm filter was placed in front of the photomultiplier to minimize possible fluorescence effects on light scattering parameters. When an analyzer was set in the horizontal direction, significant depolarized scattering was observed at either wavelength. Thus, in addition to $R_{\theta, U_{\mathrm{v}}}$ (the reduced scattering intensity at scattering angle $\theta$ for vertically polarized incident light without analyzer), $R_{\theta, H_{\mathrm{v}}}$ (the depolarized component) was measured to determine the true values of $M_{w}$ (the weight-average molecular weight), $A_{2}$ (the second virial coefficient), and $\left\langle S^{2}\right\rangle_{z}$ (the $z$-average mean-square radius of gyration) from the apparent values of $M_{w, \text { app }}, A_{2, \text { app }}$, and $\left\langle S^{2}\right\rangle_{z, \text { app}}$.

The apparatus was calibrated with pure benzene at $25^{\circ} \mathrm{C}$. The Rayleigh ratio of this liquid for unpolarized light was taken to be $46.5 \times 10^{-6}$ and $16.1 \times 10^{-6} \mathrm{~cm}^{-1}$ for 436 and $546 \mathrm{~nm}$, respectively, ${ }^{11}$ and its depolarization ratio was determined to be 0.40 for both wavelengths by the method of Rubingh and $\mathrm{Yu}^{12}$

Test solutions and NMP were optically clarified by filtration through a Millipore filter, followed by centrifugation at about $4 \times 10^{4}$ times gravity for $2 \mathrm{~h}$ (Sorvall $\mathrm{RC}-5 \mathrm{C}$ ). The solvent NMP was fractionally distilled under reduced nitrogen atmosphere after being dehydrated with calcium hydride.

The scattering intensity data obtained were analyzed according to the equations ${ }^{13-15}$

$$
\begin{gathered}
\left(K c / R_{\theta, U_{\mathrm{v}}}\right)^{1 / 2}=M_{w, \mathrm{app}}^{-1 / 2} P(\theta)_{\mathrm{app}}^{-1 / 2} \\
+A_{2, \mathrm{app}} M_{w, \mathrm{app}}^{1 / 2} P(\theta)_{\mathrm{app}}^{1 / 2} c+\cdots \\
\lim _{\substack{\theta \rightarrow 0 \\
c \rightarrow 0}} R_{\theta, H_{\mathrm{v}}} / R_{\theta, U_{\mathrm{v}}}=3 \delta /(1+7 \delta)
\end{gathered}
$$

where

$$
\begin{aligned}
P(\theta)_{\mathrm{app}}^{-1 / 2} & \\
=1+(1 / 6)\left(4 \pi n_{0} / \lambda_{0}\right)^{2}\left\langle S^{2}\right\rangle_{z, \mathrm{app}} & \sin ^{2}(\theta / 2)+\cdots \\
M_{w, \mathrm{app}} & =M_{w}(1+7 \delta) \\
\left\langle S^{2}\right\rangle_{z, \mathrm{app}} & =\left\langle S^{2}\right\rangle^{*} /(1+7 \delta) \\
A_{2, \mathrm{app}} & =A_{2} /(1+7 \delta)^{2}
\end{aligned}
$$

In these equations, $K$ is the optical constant, $c$ the polymer mass concentration, $\delta$ the optical anisotropy factor, $P(\theta)_{\mathrm{app}}$ the apparent particle scattering function, $n_{0}$ the refractive index of the solvent, $\lambda_{0}$ the wavelength of incident light in vacuum, and $\left\langle S^{2}\right\rangle^{*}$ an apparent meansquare radius of gyration. It should be noted that except for Gaussian chains, $\left\langle S^{2}\right\rangle^{*}$ differs from the true $\left\langle S^{2}\right\rangle_{z}$ unless $\delta=0$, and that a suitable model must be assumed to evaluate $\left\langle S^{2}\right\rangle_{z}$ from $\left\langle S^{2}\right\rangle^{*}$ data. $^{15}$

The specific refractive index increment $(\partial n / \partial c)$ for CTDC in NMP at $25^{\circ} \mathrm{C}$ was determined using a differential refractometer of 
the modified Schulz-Cantow type. The values of $(\partial n / \partial c)$ for 436 and $546 \mathrm{~nm}$ were 0.105 and $0.0966 \mathrm{~cm}^{3} \mathrm{~g}^{-1}$, respectively, for samples with [ $\eta$ ] (in NMP at $25^{\circ} \mathrm{C}$ ) larger than $22 \mathrm{~cm}^{3} \mathrm{~g}^{-1}$. As $[\eta]$ decreased below $20 \mathrm{~cm}^{3} \mathrm{~g}^{-1},(\partial n / \partial c)$ appreciably increased, but our light scattering measurements were confined to samples with $[\eta]>30 \mathrm{~cm}^{3} \mathrm{~g}^{-1}$.

\section{Ultracentrifugation}

Samples T360, T100, T66, T29, and T22 were investigated by sedimentation equilibrium at $25^{\circ} \mathrm{C}$, using a Beckman Model E ultracentrifuge and a Kel-F 12-mm double sector cell. The liquid column was adjusted to $1.2-1.5 \mathrm{~mm}$, and the rotor speed was chosen as $8 \times 10^{3}-2.6 \times 10^{4} \mathrm{rpm}$ depending on sample's molecular weight. Rayleigh fringe patterns read on a Nikon Shadowgraph Model 6 were converted to concentration profiles using $(\partial n / \partial c)$ (see above); for sample T22 $([\eta]=$ $\left.16.7 \mathrm{~cm}^{3} \mathrm{~g}^{-1}\right)$, the graphically interpolated $(\partial n / \partial c)$ value of $0.106 \mathrm{~cm}^{3} \mathrm{~g}^{-1}$ (at $546 \mathrm{~nm}$ ) was used. The partial specific volume $\bar{v}$ of CTDC in NMP at $25^{\circ} \mathrm{C}$ was determined to be $0.782 \mathrm{~cm}^{3} \mathrm{~g}^{-1}$ for $[\eta]>22 \mathrm{~cm}^{3} \mathrm{~g}^{-1}, 0.788 \mathrm{~cm}^{3}$ $\mathrm{g}^{-1}$ for $[\eta]=19.1 \mathrm{~cm}^{3} \mathrm{~g}^{-1}$, and $0.798 \mathrm{~cm}^{3} \mathrm{~g}^{-1}$ for $[\eta]=16.7 \mathrm{~cm}^{3} \mathrm{~g}^{-1}$ (sample T22).

The values of $M_{w}, A_{2}$, and $M_{z} / M_{w}$ (the $z$-average to weight-average molecular weight ratio) were determined by use of the equations $^{16,17}$

$$
\begin{gathered}
M_{\mathrm{SE}, \mathrm{app}}^{-1}=M_{w}^{-1}+2 A_{2} \bar{c}+\cdots \\
Q=\left(M_{w} / M_{z}\right)\left(1+2 A_{2} M_{w} \bar{c}+\cdots\right)
\end{gathered}
$$

with

$$
\begin{gathered}
M_{\mathrm{SE}, \mathrm{app}}=2 R T\left(c_{\mathrm{b}}-c_{\mathrm{a}}\right) / \\
{\left[c_{0} \omega^{2}\left(r_{\mathrm{b}}{ }^{2}-r_{\mathrm{a}}{ }^{2}\right)\left(1-\bar{v} \rho_{0}\right)\right]} \\
Q=\left(c_{\mathrm{b}}-c_{\mathrm{a}}\right)^{2} / \\
\left\{c_{0}\left(r_{\mathrm{b}}{ }^{2}-r_{\mathrm{a}}{ }^{2}\right)\left[\left(\partial c / \partial r^{2}\right)_{r=r_{\mathrm{b}}}-\left(\partial c / \partial r^{2}\right)_{r=r_{\mathrm{a}}}\right]\right\} \\
\bar{c}=\left(c_{\mathrm{a}}+c_{\mathrm{b}}\right) / 2
\end{gathered}
$$

where $r_{\mathrm{a}}$ and $r_{\mathrm{b}}$ are the radial distances from the center of rotation to the meniscus and the cell bottom, respectively, $c_{\mathrm{a}}$ and $c_{\mathrm{b}}$ are the equilibrium polymer mass concentrations at $r_{\mathrm{a}}$ and $r_{\mathrm{b}}$, respectively, $c_{0}$ is the initial polymer mass concentration, $\rho_{0}$ is the solvent density, $\omega$ is the angular velocity of the rotor, $R$ is the gas constant, and $T$ is the absolute temperature. For sample T360, $Q$ and hence $M_{z} / M_{w}$ could not be determined owing to remarkable nonideality.

\section{Viscometry}

Zero-shear viscosities of NMP solutions at $25^{\circ} \mathrm{C}$ were determined for all samples using capillary viscometers of the Ubbelohde type or four-bulb low-shear capillary viscometers (for $\left.[\eta]>6 \times 10^{2} \mathrm{~cm}^{3} \mathrm{~g}^{-1}\right)$. In evaluation of the relative viscosity for samples with $[\eta]<50$ $\mathrm{cm}^{3} \mathrm{~g}^{-1}$, the difference between the solution and solvent densities were taken into account. The Huggins plot, the Fuoss-Mead plot, and the Billmeyer plot were combined to determine $[\eta]$ and Huggins' constant $k^{\prime}$ for each sample.

\section{RESULTS}

Figures 2 and 3 show, respectively, plots of $\left(K c / R_{0, U_{\mathrm{v}}}\right)^{1 / 2}$ vs. $c$ and $P(\theta)_{\mathrm{app}}^{-1 / 2}$ vs. $\sin ^{2}(\theta / 2)$ for the indicated CTDC samples in NMP at $25^{\circ} \mathrm{C}$, where $R_{0, U_{\mathrm{v}}}$ stands for the zero-angle

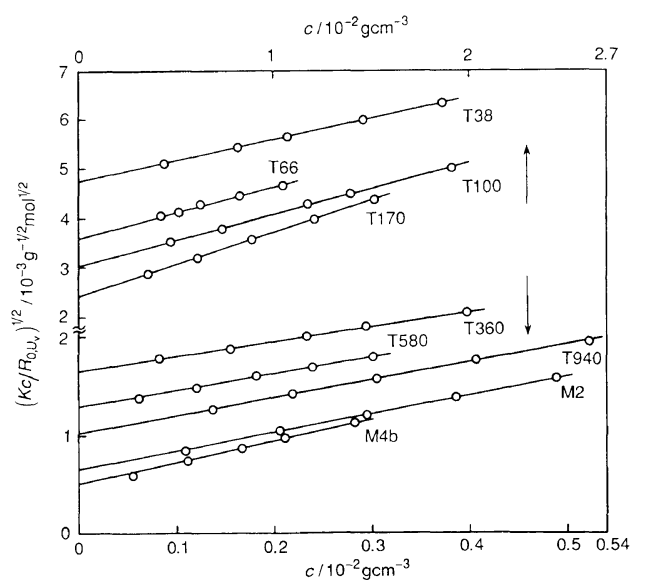

Figure 2. Plots of $\left(K c / R_{0, U_{v}}\right)^{1 / 2} v s . c$ for indicated CTDC samples in NMP at $25^{\circ} \mathrm{C}$. 


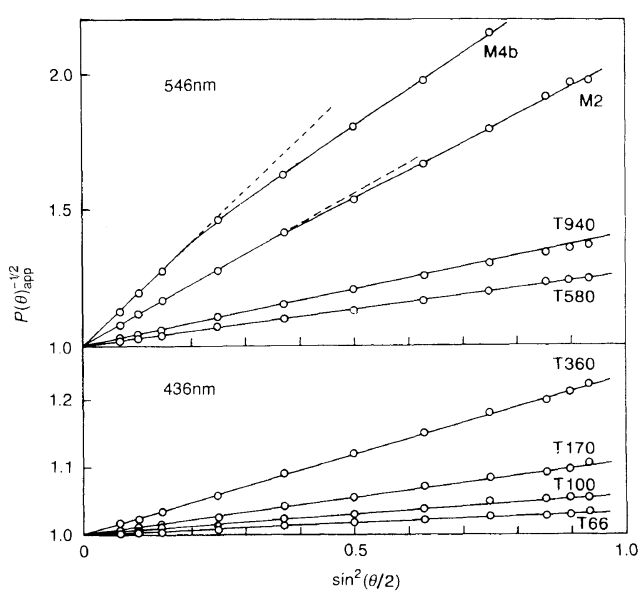

Figure 3. Plots of $P(\theta)_{\mathrm{app}}^{-1 / 2}$ vs. $\sin ^{2}(\theta / 2)$ for indicated CTDC samples in NMP at $25^{\circ} \mathrm{C}$. The dashed lines indicate the initial slopes.

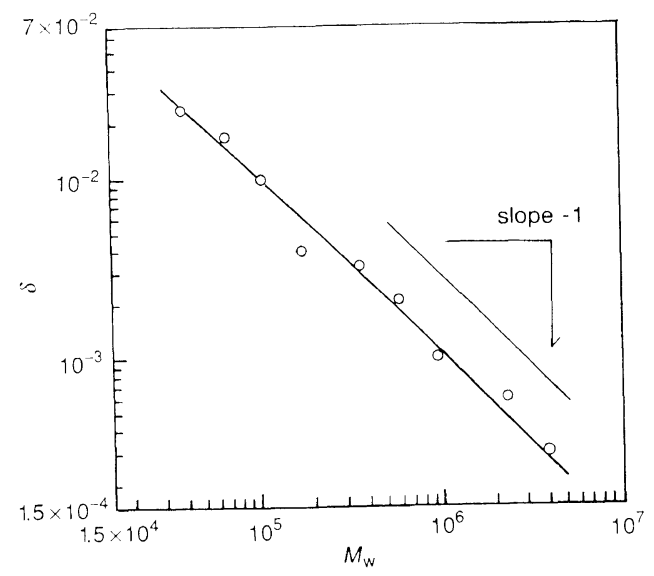

Figure 4. Molecular weight dependence of optical anisotropy factor for CTDC in NMP at $25^{\circ} \mathrm{C}$. The curve represents the theoretical values calculated from eq 16 with $q=7.8 \mathrm{~nm}, M_{\mathrm{L}}=1080 \mathrm{~nm}^{-1},|\varepsilon|=2.9$ (see the text). Its slope is about -1 for molecular weights above $10^{5}$.

value of $R_{\theta, U_{v}}$. The values of $M_{w}, A_{2}$, and $\left\langle S^{2}\right\rangle^{*}$ obtained are summarized in Table I, along with those of $\delta$ determined according to eq 2 . For samples T580, T360, T170, T100, and T66, both $M_{w}$ and $A_{2}$ determined for 436 and 546 $\mathrm{nm}$ agreed within $1 \%$, so that their averages are given in the table. We also note that the difference between the $\left\langle S^{2}\right\rangle^{*}$ values for 436 and $546 \mathrm{~nm}$ was not more than $2 \%$ for samples

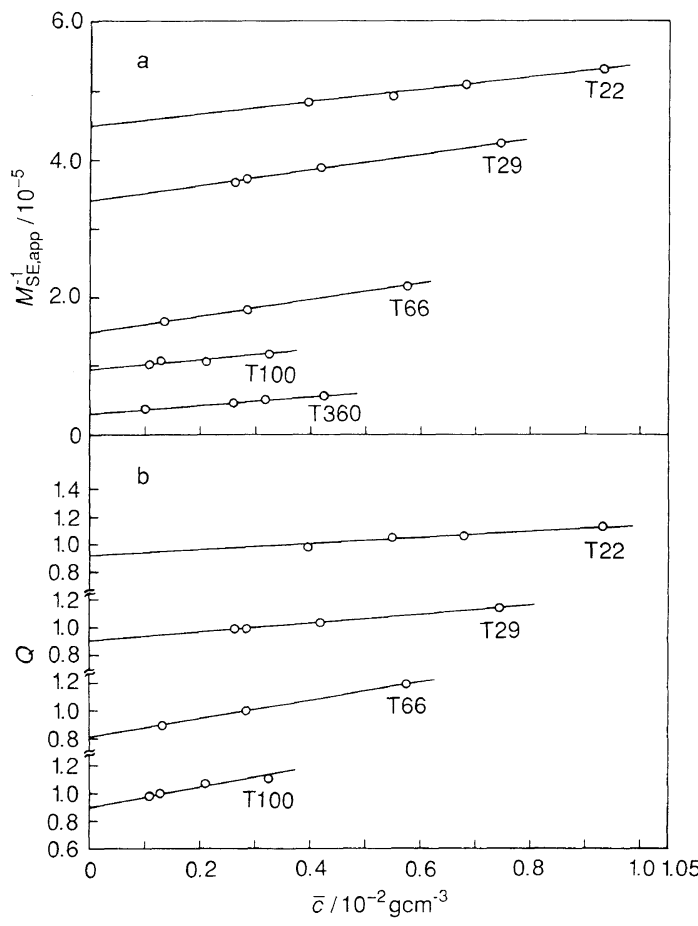

Figure 5. Plots of $M_{\mathrm{SE}, \mathrm{app}}^{-1} v s . \bar{c}$ (panel a) and $Q v s . \bar{c}$ (panel b) for indicated CTDC samples in NMP at $25^{\circ} \mathrm{C}$.

T580 and T360. Figure 4 shows that $\delta$ decreases almost linearly with $M_{w}{ }^{-1}$ in the molecular weight range studied. Though not graphically shown here, $\left\langle S^{2}\right\rangle^{*}$ and $A_{2}$ vary roughly as $M_{w}{ }^{1.1}$ and $M_{w}{ }^{-0.35}$ respectively.

Sedimentation equilibrium data are displayed in Figures $5 \mathrm{a}$ and $\mathrm{b}$, in which $M_{\mathrm{SE} \text {,app }}^{-1}$ and $Q$ are plotted against $\bar{c}$ according to eq 7 and 8 . The straight lines in the latter figure have been drawn with the aid of the $A_{2}$ values determined from the plots in the former figure. The numerical results for $M_{w}, A_{2}$, and $M_{z} / M_{w}$ are presented in Table I. The $M_{w}$ values for samples T100 and T66 are in good agreement with those from light scattering, though the agreement for sample T360 $( \pm 3 \%)$ is only moderate. The $M_{z} / M_{w}$ values indicate that samples T100, T66, T29, and T22 are fairly narrow in molecular weight distribution.

Results from viscometry are summarized in Table II, and the values of $[\eta]$ are plotted 


\section{A. Tsuboi et al.}

Table I. Results from light scattering and sedimentation equilibrium measurements on CTDC samples in NMP at $25^{\circ} \mathrm{C}$

\begin{tabular}{|c|c|c|c|c|c|c|}
\hline \multirow{2}{*}{ Sample } & \multirow{2}{*}{$M_{w} \times 10^{-4}$} & $A_{2} \times 10^{4}$ & \multirow{2}{*}{$\delta \times 10^{3}$} & \multirow{2}{*}{$M_{z} / M_{w}$} & \multirow{2}{*}{$\frac{\left\langle S^{2}\right\rangle^{*} \times 10^{12}}{\mathrm{~cm}^{2}}$} & \multirow{2}{*}{$\frac{\left\langle S^{2}\right\rangle_{z} \times 10^{12}}{\mathrm{~cm}^{2}}$} \\
\hline & & $\mathrm{molg}^{-2} \mathrm{~cm}^{3}$ & & & & \\
\hline M4b & 388 & 1.11 & 0.3 & & 101 & 101 \\
\hline M2 & 229 & 1.23 & 0.6 & & 58.8 & 58.7 \\
\hline T940 & 93.6 & 1.86 & 1.0 & & 22.4 & 22.3 \\
\hline Т580 & 58.3 & 2.16 & 2.1 & & 13.9 & 13.8 \\
\hline \multirow[t]{2}{*}{ T360 } & 35.7 & 2.60 & 3.3 & & 7.94 & 7.80 \\
\hline & $33.8^{\mathrm{a}}$ & $3.11^{\mathrm{a}}$ & & & & \\
\hline $\mathrm{T} 170$ & 17.1 & 3.39 & 4.0 & & 3.7 & $3.5_{5}$ \\
\hline \multirow[t]{2}{*}{ T100 } & 10.3 & 3.64 & 9.9 & & 2.1 & 2.0 \\
\hline & $10.5^{\mathrm{a}}$ & $3.75^{\mathrm{a}}$ & & $1.1^{\mathrm{a}}$ & & \\
\hline \multirow[t]{2}{*}{ T66 } & 6.63 & 4.32 & 17 & & $1.25^{\mathrm{b}}$ & $1.1^{\mathrm{b}}$ \\
\hline & $6.71^{\mathrm{a}}$ & $5.78^{\mathrm{a}}$ & & $1.2^{\mathrm{a}}$ & & \\
\hline T38 & 3.83 & 5.58 & 24 & & & \\
\hline $\mathrm{T} 29$ & $2.94^{\mathrm{a}}$ & $5.70^{\mathrm{a}}$ & & $1.1^{\mathrm{a}}$ & & \\
\hline $\mathrm{T} 22$ & $2.21^{\mathrm{a}}$ & $4.40^{\mathrm{a}}$ & & $1.1^{\mathrm{a}}$ & & \\
\hline
\end{tabular}

${ }^{a}$ From sedimentation equilibrium. ${ }^{b}$ Uncertainty is $\pm 15-20 \%$.

Table II. Results from viscosity measurement on CTDC samples in NMP at $25^{\circ} \mathrm{C}$

\begin{tabular}{lcc}
\hline & {$[\eta] \times 10^{-2}$} & \\
\cline { 2 - 3 } & $\mathrm{cm}^{3} \mathrm{~g}^{-1}$ & $k^{\prime}$ \\
M4mple & 9.09 & \\
M2 & 6.47 & 0.35 \\
T940 & 3.55 & 0.35 \\
T580 & 2.56 & 0.39 \\
T360 & 1.86 & 0.35 \\
T170 & 1.15 & 0.37 \\
T100 & 0.732 & 0.30 \\
T66 & 0.483 & 0.37 \\
T38 & 0.302 & 0.44 \\
T29 & 0.228 & 0.41 \\
T22 & 0.167 & 0.40 \\
\end{tabular}

double-logarithmically against $M_{w}$ in Figure 6 . The curve fitting the plotted points has a slope of about 0.95 for $M_{w}<10^{5}$ and about 0.66 for $M_{w}>2 \times 10^{5}$. The high viscosity exponent in the low molecular weight region shows the CTDC chain to be semiflexible.

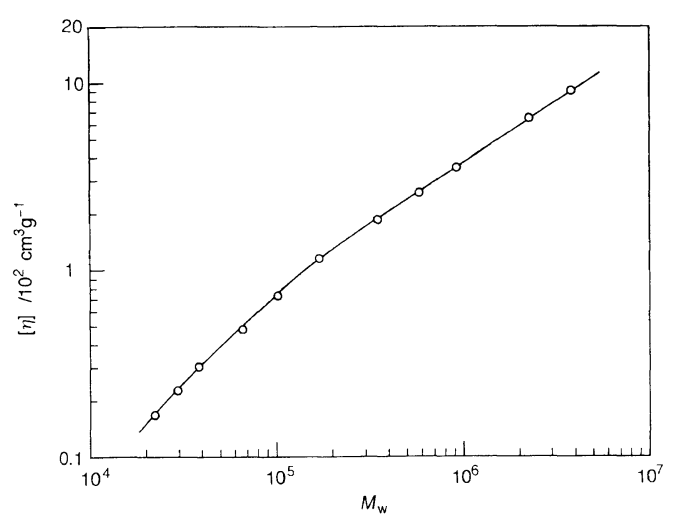

Figure 6. Molecular weight dependence of $[\eta]$ for CTDC in NMP at $25^{\circ} \mathrm{C}$.

the Kratky-Porod wormlike chain. ${ }^{9}$ The meansquare radius of gyration $\left\langle S^{2}\right\rangle$ of this chain with molecular weight $M$ is given by ${ }^{18}$

$$
\begin{aligned}
\left\langle S^{2}\right\rangle= & \left(q M / 3 M_{\mathrm{L}}\right)-q^{2}+\left(2 q^{3} M_{\mathrm{L}} / M\right) \\
& -\left(2 q^{4} M_{\mathrm{L}}{ }^{2} / M^{2}\right)\left[1-\exp \left(-M / q M_{\mathrm{L}}\right)\right]
\end{aligned}
$$

\section{DISCUSSION}

\section{Analysis of Gyration Radius Data}

We model the CTDC molecule in NMP by 


$$
\begin{aligned}
\left(M /\left\langle S^{2}\right\rangle\right)^{1 / 2}= & \left(3 M_{\mathrm{L}} / q\right)^{1 / 2} \\
& +q^{1 / 2}\left(3 M_{\mathrm{L}}\right)^{3 / 2} /(2 M)
\end{aligned}
$$

provided that $M / 2 q M_{\mathrm{L}}$ (the Kuhn segment number) $>2$.

If the chain has cylindrically symmetric polarizabilities and has a Kuhn number greater than 2, Nagai's theory ${ }^{15}$ for $\left\langle S^{2}\right\rangle^{*}$ is written in a good approximation as ${ }^{20}$

$$
\left\langle S^{2}\right\rangle^{*}=\left\langle S^{2}\right\rangle-f_{U_{v}}
$$

with

$$
\begin{aligned}
f_{U_{\mathrm{v}}}= & \left(4 q^{2} / 45\right)\left\{\varepsilon \left[1-\left(8 q M_{\mathrm{L}} / 3 M\right)\right.\right. \\
& \left.+\left(26 q^{2} M_{\mathrm{L}}^{2} / 9 M^{2}\right)\right] \\
& -\left(23 \varepsilon^{2} q M_{\mathrm{L}} / 126 M\right) \\
& \left.\times\left[1-\left(103 q M_{\mathrm{L}} / 69 M\right)\right]\right\}
\end{aligned}
$$

Here, $\varepsilon$ is the polarizability parameter related to $\delta$ by ${ }^{15}$

$$
\begin{aligned}
\delta= & \left(2 \varepsilon^{2} q M_{\mathrm{L}} / 135 M\right) \\
& \times\left\{1-\left(q M_{\mathrm{L}} / 3 M\right)\left[1-\exp \left(-3 M / q M_{\mathrm{L}}\right)\right]\right\}
\end{aligned}
$$

For the cylindrically symmetric chain considered here, $\varepsilon$ is defined by $3\left(\alpha_{1}-\alpha_{2}\right) /\left(\alpha_{1}+\right.$ $2 \alpha_{2}$ ), with $\alpha_{1}$ and $\alpha_{2}$ being the longitudinal and transverse polarizabilities per unit contour length, but its value intrinsic to the molecule cannot be derived directly from $\delta$ or $R_{0, H_{\mathrm{v}}}$ data, because the measured (total) $R_{0, H_{v}}$ generally contains a substantial contribution from the collision-induced component ${ }^{21-23}$ and also because $\varepsilon$ in solution is subjected to the effect of internal field ${ }^{24}$; correction for these effects may lower $|\varepsilon|$ by a factor of 2 . In the present work, we regard $\varepsilon$ as an apparent polarizability parameter independent of molecular weight. We also note that although eq 16 does not always require the cylindrical symmetry of polarizabilities, ${ }^{25}$ no such simple relation as eq 14 is as yet known for a generalized wormlike chain (see ref 25).

As may be seen from eq 12 through 16 , three parameters $q, M_{\mathrm{L}}$, and $\varepsilon$ must be known in order to evaluate the true $\left\langle S^{2}\right\rangle_{z}$ from $\left\langle S^{2}\right\rangle^{*}$ data. We determined these parameters and $\left\langle S^{2}\right\rangle_{z}$ at one time from the data of $\left\langle S^{2}\right\rangle^{*}, \delta$, and $M_{w}$ in Table $I$ by the following iteration method. ${ }^{20}$

First, approximate values of $M_{\mathrm{L}}$ and $q$ were estimated from the intercept and slope of the $\left(M_{w} /\left\langle S^{2}\right\rangle^{*}\right)^{1 / 2} v s . M_{w}{ }^{-1}$ plot shown in Figure $7 \mathrm{a}$, in which the downward deviations of the two plotted points for small $M_{w}{ }^{-1}$ from the indicated straight line have been regarded as due to intramolecular excluded-volume effects. The approximate values of $M_{\mathrm{L}}$ and $q$ were then used to find $\varepsilon$ which allows eq 16 to best fit the experimental $\delta$ vs. $M_{w}$ relation in Figure $4 ; \varepsilon$ for CTDC was taken to be negative on the basis of flow birefringence data ${ }^{26,27}$ for cellulose tris(phenylcarbamate) in various solvents. With the values of $M_{\mathrm{L}}, q$, and $\varepsilon$ thus estimated, $f_{U_{\mathrm{v}}}$ in eq 14 was calculated to obtain first approximate values of $\left\langle S^{2}\right\rangle_{z}$ as a function of $M_{w}$. When this procedure was iterated twice,

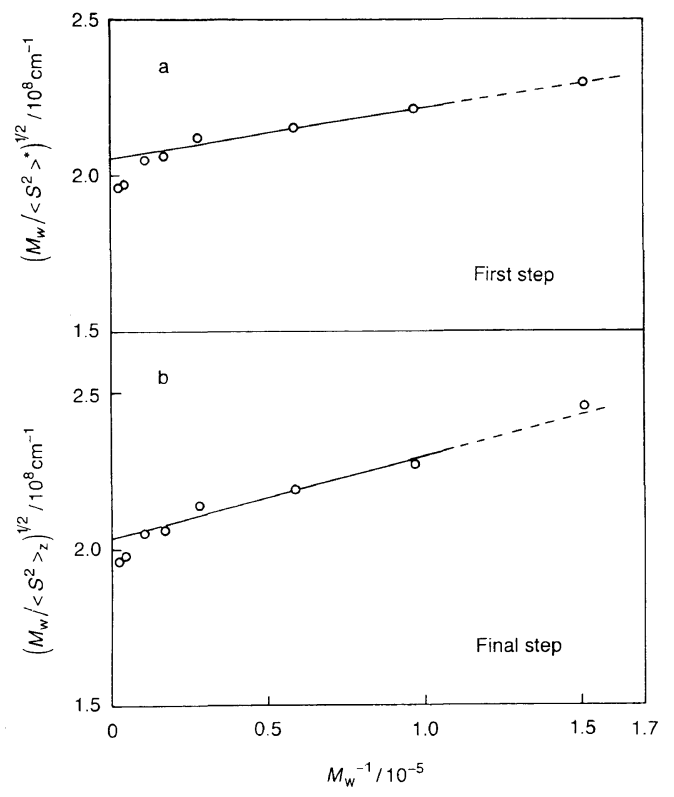

Figure 7. Plots of $\left(M_{w} /\left\langle S^{2}\right\rangle^{*}\right)^{1 / 2}$ vs. $M_{w}{ }^{-1}$ (panel a) and $\left(M_{w} /\left\langle S^{2}\right\rangle_{z}\right)^{1 / 2} v s . M_{w}{ }^{-1}$ (panel b) for CTDC in NMP at $25^{\circ} \mathrm{C}$. Since $\left(M_{w} /\left\langle S^{2}\right\rangle^{*}\right)^{1 / 2}$ and $\left(M_{w} /\left\langle S^{2}\right\rangle_{z}\right)^{1 / 2}$ at $M_{w}{ }^{-1}=$ $1.51 \times 10^{-5}$ contain considerable uncertainty, the best fit straight lines around this $M_{w}{ }^{-1}$ are indicated by the dashed ones. 
the following convergent parameter values were obtained: $q=7.8( \pm 0.6) \mathrm{nm}, M_{\mathrm{L}}=1080$ $( \pm 60) \mathrm{nm}^{-1}, \varepsilon=-2.9( \pm 0.2)$ (the values of $\left\langle S^{2}\right\rangle_{z}$ are presented in the last column of Table I). The $\left(M_{w} /\left\langle S^{2}\right\rangle_{z}\right)^{1 / 2} v s . M_{w}{ }^{-1}$ plot in the final step is shown in Figure $7 \mathrm{~b}$. The curve in Figure 4 actually represents the $\delta$ values calculated from eq 16 with the above parameter values.

In Figure 8, the experimental $\left\langle S^{2}\right\rangle_{z}$ values are compared with the theoretical curve computed from eq 12 with $q=7.8 \mathrm{~nm}$ and $M_{\mathrm{L}}=$ $1080 \mathrm{~nm}^{-1}$. The agreement is quite satisfactory for $M_{w}$ below $10^{6}$. Excluded-volume effects appear for $M_{w}>10^{6}$, but they remain small even at the highest molecular weight studied. This behavior seems to be typical of semiflexible chains. With regard to polydispersity effects on the model parameters, the following remarks may be in order.

Since in the molecular weight range examined, $\left\langle S^{2}\right\rangle$ is determined substantially by the first two terms $\left(q M / 3 M_{\mathrm{L}}\right.$ and $\left.-q^{2}\right)$ on the right hand side of eq 12 , the $M_{\mathrm{L}}$ value estimated from $\left\langle S^{2}\right\rangle_{z}$ should be smaller roughly by a factor of $M_{z} / M_{w}$ than the "polydispersity-free value," while $q$ may not greatly be affected by polydispersity. If $M_{z} / M_{w}$ is taken as 1.1 (see Table I), $M_{L}$ is obtained as $1190 \mathrm{~nm}^{-1}$, which

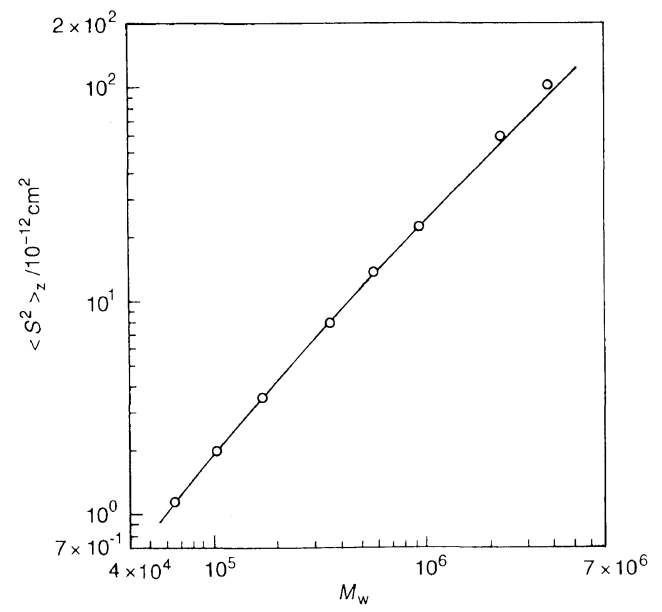

Figure 8. Experimental $\left\langle S^{2}\right\rangle_{z}$ for CTDC in NMP compared with the theoretical curve (eq 12) for the wormlike chain with $q=7.8 \mathrm{~nm}$ and $M_{\mathrm{L}}=1080 \mathrm{~nm}^{-1}$. in turn gives $\varepsilon=-2.8$. Note that $\varepsilon^{2} M_{\mathrm{L}}$ should be essentially free from polydispersity since $\delta$ is roughly proportional to $M_{w}{ }^{-1}$ in the molecular weight range studied (see eq 16). We have recalculated $\left\langle S^{2}\right\rangle_{z}$ from eq 14 and 15 with $\varepsilon=-2.8 \quad\left(q=7.8 \mathrm{~nm}\right.$ and $M_{\mathrm{L}}=1080$ $\mathrm{nm}^{-1}$ ) and found that the correction of $\varepsilon$ does not alter the $\left\langle S^{2}\right\rangle_{z}$ values in Table I.

\section{Analysis of Viscosity Data}

The theory of Yoshizaki et al. ${ }^{28}$ for $[\eta]$ of a wormlike touched-bead chain contains three parameters, $q, M_{\mathrm{L}}$, and $d_{\mathrm{b}}$ (the bead diameter). It was found that the present $[\eta]$ data allow only two of these three parameters to be determined uniquely. Thus with the $q$ value of $7.8 \mathrm{~nm}$ determined above from $\left\langle S^{2}\right\rangle_{z}$, we searched for $d_{\mathrm{b}}$ and $M_{\mathrm{L}}$ which lead to the closest agreement between the theory of Yoshizaki et al. and our $[\eta]$ data.

Figure 9 shows that the $[\eta]$ data for $M_{w}<10^{6}$ are closely fitted by the theoretical curve for $q=7.8 \mathrm{~nm}, M_{\mathrm{L}}=1140 \mathrm{~nm}^{-1}$, and $d_{\mathrm{b}}=3.9 \mathrm{~nm}$. The substantial agreement between this $M_{\mathrm{L}}$ and the value $1190 \mathrm{~nm}^{-1}$ from $\left\langle S^{2}\right\rangle$ allows us to conclude that the present data for $\left\langle S^{2}\right\rangle_{z}, \delta$, and $[\eta]$ are explained consistently by the theories ${ }^{15,18,28}$ for the Kratky-Porod chain. The upward deviations of the data points for the two (or three) highest molecular weight

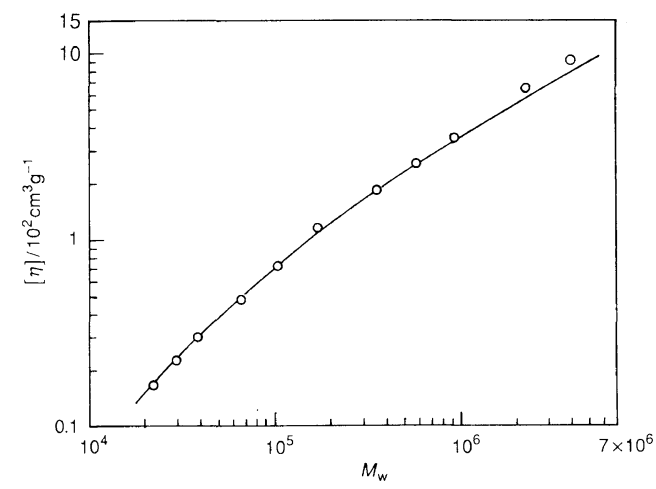

Figure 9. Comparison of the measured $[\eta]$ for CTDC in NMP with the theoretical curve computed from the theory of Yoshizaki et al. ${ }^{28}$ for the wormlike touched-bead chain with $q=7.8 \mathrm{~nm}, M_{\mathrm{L}}=1140 \mathrm{~nm}^{-1}$, and $d_{\mathrm{b}}=3.9 \mathrm{~nm}$. 
samples (i.e., for $M_{w}>6 \times 10^{5}-10^{6}$ ) may be ascribed to excluded-volume effects on $[\eta]$. This finding is consistent with what we have seen above for $\left\langle S^{2}\right\rangle_{z}$. The critical molecular weight $\left(6 \times 10^{5}-10^{6}\right)$, above which volume effects become experimentally visible, corresponds to a Kuhn segment number of $30-50$, which is comparable to the earlier estimate ${ }^{29}$ for stiff chains.

Recently, Hayashi et al. ${ }^{30}$ showed with $[\eta]$ data for sodium hyaluronate in 0.2 or $0.5 \mathrm{M}$ aqueous sodium chloride $(q=4.1$ or $4.2 \mathrm{~nm})$ that, without explicit consideration of volume effect in data analysis, $q$ is appreciably overestimated. This is because stiffness and excluded-volume effects can lead to similar molecular weight dependences of $[\eta]$ (probably $\left\langle S^{2}\right\rangle$ as well) in a certain narrow range of molecular weight. However, we have found that for CTDC stiffer than Na hyaluronate (at high ionic strength), virtually no alteration is needed for the model parameter values from $\left\langle S^{2}\right\rangle_{z}$ and $[\eta]$ even when excluded-volume effects are explicitly taken into account in the framework of the Yamakawa-StockmayerShimada theory ${ }^{31-33}$ for wormlike or helical wormlike chains. The point is that for an appropriate value of the excluded-volume strength parameter this theory predicts volume effects small enough for $M_{w}<6 \times 10^{5}$ but appreciable for $M_{w}>10^{6}$. Detailed discussions on the volume effects will be given elsewhere when more $\left\langle S^{2}\right\rangle_{z}$ and $[\eta]$ data, especially for high molecular weights, accumulate.

\section{Molecular Characteristics}

The estimated $M_{\mathrm{L}}$ values $\left(1190 \mathrm{~nm}^{-1}\right.$ from $\left\langle S^{2}\right\rangle$ and $1140 \mathrm{~nm}^{-1}$ from [ $\left.\left.\eta\right]\right)$ along with the molar mass 603.7 per residue yield 0.52 ( \pm $0.01) \mathrm{nm}$ for the monomeric length $h$ along the contour of the CTDC chain. This $h$ value is in substantial agreement with the pitch per residue $(0.51 \mathrm{~nm})$ of the left-handed three-fold helix proposed by Zugenmaier and $\mathrm{Vogt}^{34}$ for the crystalline structure of cellulose tris(phenylcarbamate) (CTC). However, this agreement does not necessarily demonstrate that the CTDC chain in NMP locally maintains the helical conformation (or similar conformation), since our $h$ value is equally close to the monomeric projection for the trans conformation of cellulose $(0.518 \mathrm{~nm})^{35}$ or cellulose triacetate $(0.522 \mathrm{~nm})^{36}$ in the crystalline state. The difference in $h$ between the trans and helical conformations is too small to be distinguished by solution work. The reported $M_{\mathrm{L}}$ values for $\mathrm{CTC}^{37,38}$ and other derivatives ${ }^{39,40}$ in dilute solution indeed give $h$ values of about $0.52 \mathrm{~nm}$, as is the case for CTDC.

The persistence length $7.8 \mathrm{~nm}$ for CTDC in NMP may also be considered typical of cellulose derivatives in that most of the reported $q$ values ${ }^{37-43}$ are in the range between 3 and $17 \mathrm{~nm}$. For CTC, which has been studied most extensively by a variety of solution techniques, ${ }^{26,27,37,44-46} q$ varies with solvent conditions from 3 to $14 \mathrm{~nm}$. Because of this large variation in $q$, comparison between the values for CTDC in NMP and CTC in other solvents is irrelevant. Evidently, experimental determination of $q$ for both CTDC and CTC under the same solvent condition is needed to see whether or not the chain stiffness is influenced by the presence of 3,5-dimethyl groups on the phenylcarbamate substituent.

Our $d_{\mathrm{b}}$ value $3.9 \mathrm{~nm}$ based on the theory of Yoshizaki et $a .^{28}$ corresponds to a cylinder diameter $d$ of $2.9 \mathrm{~nm}$ (see ref 28), which appears too large compared to those $(1.15-1.3 \mathrm{~nm})$ reported by Dan̆helka et $a .^{37}$ for CTC in various solvents even if the contribution from 3,5-dimethyl groups to $d$ is taken into consideration. However, the smaller $d$ values of this group must be accepted with reservation since they are based on the assumption that the hydrodynamic volume occupied by one gram of wormlike cylinders is equal to $\bar{v}$; note that this assumption does not always hold. ${ }^{38}$ Our $d$ value of $2.9 \mathrm{~nm}$ is larger than the maximal diameter of $2.5 \mathrm{~nm}$ estimated from the chemical structure of CTDC. If literally taken, this difference implies that NMP molecules solvate 


\section{A. Tsubol et al.}

onto the CTDC chain to some extent.

As found in this work, the CTDC chain in NMP is optically anisotropic. Interestingly, none of the previous light scattering studies detected such anisotropy for CTC. Nonetheless, flow birefringence data ${ }^{27}$ show that among other cellulose derivatives so far studied, CTC has the largest, negative excess polarizability $\Delta \alpha\left(=\alpha_{1}-\alpha_{2}\right)$ though the magnitude depends on the kind of solvent. ${ }^{26,27}$ All these experimental facts combined together suggest that the CTDC molecule should have an extraordinary large, negative $\Delta \alpha$ compared to other cellulose derivatives and that there must exist an unmistakable difference in the relative orientation of phenylene rings between CTDC and CTC. Such a difference arising from the presence or absence of 3,5-dimethyl groups may have something to do with the higher optical resolution capacity of $\mathrm{CTDC}^{2,4}$ than that of CTC in chromatographic separation of racemic compounds. According to Okamoto et al., ${ }^{2}$ phenyl groups play an important part in the chiral recognition of cellulose carbamate derivatives.

\section{CONCLUSIONS}

We prepared narrow-distribution fractions of cellulose tris(3,5-dimethylphenylcarbamate) covering a range in $M_{w}$ from $2 \times 10^{4}$ to $4 \times 10^{6}$ and investigated them by light scattering, sedimentation equilibrium, and viscometry with 1-methyl-2-pyrrolidone at $25^{\circ} \mathrm{C}$ as the solvent. The major findings and conclusions from this work may be summarized as follows.

1. The polymer exhibits pronounced optical anisotropy differing from other cellulose derivatives. 2. The molecular weight dependences of $\left\langle S^{2}\right\rangle_{z}, \delta$ (the optical anisotropy factor), and $[\eta]$ are described quantitatively by the known expressions ${ }^{15,18,28}$ for the KratkyPorod wormlike chain with a persistence length of $7.8 \mathrm{~nm}$, a monomeric projection of $0.52 \mathrm{~nm}$, an apparent polarizability parameter of -2.8 , and a bead diameter of $3.9 \mathrm{~nm}$. 3. Excluded- volume effects on $\left\langle S^{2}\right\rangle_{z}$ and $[\eta]$ become experimentally visible when $M_{w}$ exceeds $10^{6}$, but they remain small even at $M_{w}=4 \times 10^{6}$, the highest molecular weight studied.

Acknowledgments. We thank Professor Y. Okamoto of Nagoya University for his interest in this work and Dr. H. Kojima of Daicel Co. for providing 3,5-dimethylphenylisocyanate. This work was supported by a Grant-in-Aid for Scientific Research (06403027) from the Ministry of Education, Science, and Culture of Japan.

\section{REFERENCES}

1. Y. Okamoto, M. Kawashima, and K. Hatada, J. Am. Chem. Soc., 106, 5357 (1984).

2. Y. Okamoto, M. Kawashima, and K. Hatada, $J$. Chromatogr., 363, 173 (1986).

3. Y. Okamoto, R. Aburatani, Y. Kaida, and K. Hatada, Chem. Lett., 1125 (1988).

4. Y. Okamoto and R. Aburatani, Polym. News, 14, 295 (1989).

5. W. Burchard and E. Husemann, Makromol. Chem., 44 46, 358 (1961).

6. V. P. Shanbhag, Ark. Kemi, 29, 1 (1968).

7. W. Burchard, Polymer, 11, 467 (1969).

8. J. T. Guthrie, M. B. Huglin, R. W. Richards, V. I. Shah, and A. H. Simpson, Eur. Polym. J., 11, 527 (1975).

9. O. Kratky and G. Porod, Rec. Trav. Chim., 68, 1106 (1949).

10. D. Henley, Ark. Kemi, 18, 327 (1961).

11. Dj. Deželić and J. Vavra, Croat. Chem. Acta, 38, 35 (1966).

12. D. N. Rubingh and H. Yu, Macromolecules, 9, 681 (1976).

13. G. C. Berry, J. Chem. Phys., 44, 4550 (1966).

14. H. Yamakawa, "Modern Theory of Polymer Solutions,"'Harper \& Row, New York, N.Y., 1971.

15. K. Nagai, Polym. J., 3, 67 (1972).

16. H. Fujita, "Foundations of Ultracentrifugal Analysis," Wiley, New York, N.Y., 1975.

17. T. Norisuye, T. Yanaki, and H. Fujita, J. Polym. Sci., Polym. Phys. Ed., 18, 547 (1980).

18. H. Benoit and P. Doty, J. Phys. Chem., 57, 958 (1953).

19. H. Murakami, T. Norisuye, and H. Fujita, Macromolecules, 13, 345 (1980).

20. K. Sakurai, K. Ochi, T. Norisuye, and H. Fujita, Polym. J., 16, 559 (1984).

21. C. W. Carlson and P. J. Flory, J. Chem. Soc., Farady Trans. 2, 73, 1505 (1977). 
22. U. W. Suter and P. J. Flory, J. Chem. Soc., Farady Trans. 2, 73, 1521 (1977).

23. T. Konishi, T. Yoshizaki, J. Shimada, and H. Yamakawa, Macromolecules, 22, 1921 (1989).

24. P. J. Flory, "Statistical Mechanics of Chain Molecules," Wiley, New York, N.Y., 1969.

25. H. Yamakawa, M. Fujii, and J. Shimada, J. Chem. Phys., 71, 1611 (1979).

26. J. W. M. Noordermeer, R. Daryanani, and H. Janeschitz-Kriegl, Polymer, 16, 359 (1975).

27. V. N. Tsuetkov and L. N. Andreeva, Adv. Polym. Sci., 39, 95 (1981).

28. T. Yoshizaki, I. Nitta, and H. Yamakawa, Macromolecules, 21, 165 (1988).

29. T. Norisuye and H. Fujita, Polym. J., 14, 143 (1982).

30. K. Hayashi, K. Tsutsumi, F. Nakajima, T. Norisuye, and A. Teramoto, Macromolecules, 28, 3824 (1995).

31. H. Yamakawa and W. H. Stockmayer, J. Chem. Phys., 57, 2843 (1972).

32. H. Yamakawa and J. Shimada, J. Chem. Phys., 83, 2607 (1985).

33. J. Shimada and H. Yamakawa, J. Chem. Phys., 85, 591 (1986).

34. P. Zugenmaier and U. Vogt, Makromol. Chem., 184,
1749 (1983).

35. Y. Takahashi, Macromolecules, 24, 3968 (1991).

36. H. Steinmeier and P. Zugenmaier, Carbohydr. Res., 164, 97 (1987).

37. J. Dan̆helka, M. Netopilík, and M. Bohdanecký, $J$. Polym. Sci., B, Polym. Phys., 25, 1801 (1987).

38. T. Norisuye, Prog. Polym. Sci., 18, 543 (1993).

39. H. Yamakawa and M. Fujii, Macromolecules, 7, 128 (1974).

40. A. Takada, T. Fukuda, J. Watanabe, and T. Miyamoto, Macromolecules, 28, 3394 (1995).

41. G. Conio, E. Bianchi, A. Ciferri, A. Tealdi, and M. A. Aden, Macromolecules, 16, 1264 (1983).

42. G. V. Laivins and G. D. Gray, Macromolecules, 18, 1746 (1985).

43. P. Sixou and A. Ten Bosh, "Cellulose: Structure, Modification and Hydrolysis," R. A. Young and R. M. Rowell, Ed., Wiley, New York, N.Y., 1986.

44. A. K. Gupta, E. Marchal, and W. Burchard, Macromolecules, 8, 843 (1975).

45. W. Sutter and W. Burchard, Makromol. Chem., 179, 1961 (1978).

46. F. Fried, G. M. Searby, M. J. Seurin-Vellutini, S. Dayan, and P. Sixou, Polymer, 23, 1755 (1982). 